\title{
Germaine Guèvremont, la dame des îles
}

\author{
David Décarie \\ Université de Moncton
}

Ce dossier est dédié à la mémoire d'Yvan et Françoise Lepage

Publié en 1945, Le Survenant de Germaine Guèvremont, qui raconte l'arrivée d'un étranger dans la famille Beauchemin et dans la petite communauté paysanne du Chenal du Moine a, dès sa première réception (Annette Hayward le montre dans ce numéro), été associé au roman du terroir. Encore aujourd'hui, il 
est assez commun de lire que cette œuvre a marqué à la fois l'apogée du roman du terroir et son chant du signe.

À bien y regarder, pourtant, la terre et le travail de la terre y occupent une place somme toute restreinte. L'équivoque débute dès le recueil de nouvelles qui ouvre le Cycle du Survenant, En pleine terre, paru en 1942, et qui décrit à peu près tous les aspects de la vie paysanne... sauf le travail de la terre. La page couverture dessinée par Maurice Petitdidier pour l'édition de 1955 illustre le caractère double, à la fois terrien et aquatique de l'œuvre : le premier plan, en contradiction avec le titre, est en effet occupé par trois canards s'envolant d'une étendue d'eau. Cette dualité se retrouve également dans le nom du principal lieu du cycle : le Chenal du Moine, un chenal étant un « passage ouvert à la navigation entre un port, une rivière ou un étang et la mer, entre des rochers, des îles, dans le lit d'un fleuve ». Comme les œuvres de Gabrielle Roy, de Félix-Antoine Savard ou de Roger Lemelin, mais aussi celles des pionnières du roman au féminin aujourd'hui moins connues - Laetitia Filion, Marie Lefranc, Michelle le Normand, Adrienne Maillet - le Cycle du Survenant est une œuvre de transition. Née dans le terroir, dans la tradition, dans l'univers sévère du patriarche Didace Beauchemin, l'œuvre de Guèvremont est pourtant tout entière tournée vers les îles et le fleuve, fenêtres ouvertes sur le « vaste monde », et vers le mystère de tout ce qui peut en survenir.

Dans l'émission «Femme d'aujourd'hui » du 24 février 1976 produite par Radio-Canada en hommage à Germaine Guèvremont, celle-ci fut poétiquement surnommée la dame des îles. La romancière, en effet, habita, de 1920 à 1935, la région de Sorel et se fit construire, en 1957, un chalet dans l'îlette communément appelée «au Pé » ou « O. P. » : «Pour atteindre 
cette île, il faut traverser le pont qui joint la terre ferme à l'îleaux-Fantômes, puis de là, monter en chaloupe. C'est au moyen d'une solide corde qu'on fait avancer l'embarcation jusqu'à l'île O.P. ${ }^{1} \gg$

Mais c'est bien sûr avant tout par son travail d'écrivaine que Guèvremont se mérita ce titre. Avec une constance et une fidélité à toute épreuve, elle fit découvrir à un large public la région de Sorel, des îles de Sorel et du Chenal du Moine. Elle s'intéressa d'abord à ce coin de pays comme journaliste, au Courrier de Sorel (peut-être dès 1926) et comme correspondante à The Gazette (à partir de novembre 1928), puis dans des textes de fiction. D'avril 1939 jusqu'à octobre 1940, Guèvremont publia un roman feuilleton, Tu seras journaliste, qui racontait sa découverte de Sorel et du métier de journaliste. Elle écrivit de plus, dès la première parution de la revue Paysana en mars 1938, la première ébauche de son cycle, une nouvelle intitulée « Les survenants » qui met en scène des paysans du Chenal du Moine. Elle publia à Paysana de nombreuses autres nouvelles qui furent réunies en volume, en 1942, sous le titre d'En pleine terre ${ }^{2}$. En 1945, elle donna une suite au recueil de nouvelles en publiant Le Survenant qui connut un succès considérable et qui fut suivi en 1947 de Marie-Didace. Dans les années suivantes, elle approfondit et continua son cycle sous forme de radioroman (CBF 1952-1955, CKVL 1962-1965) puis de téléroman (CBFT 1954-1960). La matière d'un troisième roman que prévoyait écrire l'auteure a donné lieu aux deux dernières séries télévisées (Marie-

\footnotetext{
1 Anonyme, « Dix-sept ans après. Le Survenant, où il a été, et ce qu'il a fait », le Petit journal, 22 novembre 1959, p. 112.

2 Selon Guèvremont, plusieurs de ces nouvelles furent d'abord publiées dans Le courrier de Sorel en 1933 et 1934.
} 
Didace et Le Survenant, CBFT 1958-1960) dans lesquelles Guèvremont introduisait une nouvelle génération de personnages. Enfin, peu avant son décès, le 21 août 1968, elle écrivit le scénario du film L'adieu aux îles qui fut réalisé par Jean-Paul Fugère et diffusé le 4 juin 1968. La dame des îles laisse ainsi deviner que son œuvre est au moins aussi insulaire et aquatique que terrienne, comme en témoignent les nombreux passages de ses trois œuvres qui se déroulent sur l'eau. Ajoutons que l'insularité des îles de Sorel est une insularité particulière et qui a peu à voir avec l'archétype habituel de lîle (l'Île d'Orléans de Félix Leclerc, par exemple). La pluralité des îles de Sorel rend par exemple impossible la clôture et le retrait traditionnellement associé à l'imaginaire de l'île: l'identité des îles est multiple, composite, ouverte. Le caractère maritime et aquatique - important dans tout imaginaire de l'île - est encore renforcé dans le cas des îles de Sorel par cette fragmentation du territoire : on peut faire le tour d'une île à pied, mais le tour des îles ne peut se faire que sur l'eau, en canot ou en bateau. Les îles de Sorel forment enfin une géographie variable, elles changent selon les saisons et les lunes: l'hiver relie toutes les îles entre elles par un pont de glace, le printemps apporte le "tue-monde» des inondations. La diversité et l'ouverture de ce paysage situé entre deux rives sont à l'image de l'œuvre de Germaine Guèvremont. Les quatre études réunies ici s'intéressent à la passeuse («Personne qui conduit un bac, un bateau, une barque pour traverser un cours d'eau. Personne qui fait passer clandestinement une frontière, traverser une zone interdite, etc. ») que fut la dame des îles. Les passages, réels et symboliques, abondent dans le Cycle du Survenant. La figure du psychopompe («Qui conduit les âmes des morts ») ou celle du nocher des enfers apparaît en filigrane 
dans plusieurs passages. Dans «Un petit Noël» (En pleine terre), ce rôle revient au mari de Marie-Amanda, Ludger Aubuchon, qui doit traverser en chaloupe le fleuve à moitié gelé afin de mener une habitante de l'île de Grâce récemment décédée vers son dernier repos. La venue de Venant, de même, permet à Didace de faire le deuil de son fils Éphrem, mort noyé après que son canot se fut renversé, et d'accepter sa propre mort, survenue à la suite d'une crise cardiaque lors d'une partie de chasse entreprise elle aussi en canot :

Le père Beauchemin avait eu une attaque d'angine, au milieu de la nuit. Les chasseurs l'avaient couché sur la paille, au fond de son canot, à l'abri du vent, sous le prélart de chasse. Mais ils avaient dû attendre la clarté pour sortir de l'affût et retrouver leur chemin parmi les chenaux. Au jour, les appelants levés et le canot attaché à leur embarcation, l'orage avait éclaté. Vent devant, le canot à la touée, ils avaient lentement remonté le courant ${ }^{3}$.

Mais le «trafic littéraire » de Guèvremont, les quatre études le montrent, prend de multiples formes : il se fait à la fois aux plans culturel, identitaire, temporel et générique. Étudiant la correspondance de Germaine Guèvremont avec William Arthur Deacon, Yvan Lepage montre que Guèvremont, par sa connaissance de l'anglais et par son amitié avec Deacon, joua un rôle important dans la reconnaissance de la littérature québécoise au Canada anglais. Cet article, rempli de renseignements utiles aux chercheurs et qui offre une vue d'ensemble de la carrière littéraire de cette auteure, est un des tout derniers textes rédigés par Yvan Lepage, grand érudit et spécialiste de l'œuvre de Guèvremont.

3 Marie-Didace, édition critique par Yvan G. Lepage, Montréal, Presses de l'Université de Montréal, coll. "Bibliothèque du Nouveau Monde », [1947] 1996, p. 296. 
Annette Hayward s'intéresse quant à elle à l'identité hybride de cet étranger que Guèvremont fit advenir dans son roman au Chenal du Moine, et à mieux comprendre la surprenante décision qu'elle prit en 1968 de supprimer le passage où celle-ci était mise de l'avant. En examinant l'évolution du contexte de production et de réception entre 1945 et 1968, puis en faisant le lien avec l'époque contemporaine, elle suggère que le moment est venu de revoir la politique éditoriale consistant à occulter cet aspect de l'œuvre de Guèvremont. Un article séparé fournit ensuite une bibliographie commentée consacrée à la réception du Survenant en 1945.

Andréa King étudie le rôle de pionnière joué par Guèvremont dans l'écriture au féminin au Québec et souligne dans l'œuvre les traces de l'angoisse causée par le choix de devenir écrivain, un métier plus ou moins licite pour une femme à l'époque. Elle s'intéresse ainsi à l'ambivalence de Guèvremont face à l'écriture, une ambivalence qui se devine en filigrane dans l'œuvre, notamment dans la grossesse problématique de Phonsine et dans la figure de la revenante.

David Décarie analyse enfin la question de l'intermédialité en examinant les opérations de traduction ou de transformation des figures de style qui ont lieu entre le roman Le Survenant et ses transpositions à la radio et à la télévision. Il montre notamment l'importance que prête Guèvremont à la conservation du registre symbolique d'un média à l'autre, remettant ainsi en question la frontière rigide que dresse parfois la critique entre littérature et «paralittérature ». 
L'œuvre de Guèvremont reflète une époque complexe où le Québec était déchiré par des tensions idéologiques très fortes. Elle-même n'échappait pas à ces tensions, comme en témoigne le traitement des personnages féminins, analysée par Andrea King, ou son rapport au contexte discursif dominant au Québec au sortir de la Deuxième Guerre mondiale, comme le montre Annette Hayward. Profondément en harmonie avec son temps, l'écrivaine s'adaptera aussi aux nouveaux médias, comme le mettent en évidence les transformations que relève David Décarie dans les figures de style lors de l'adaptation des romans à la radio puis à la télévision. La réception extraordinaire du Survenant auprès de publics divers au fil des années témoigne du génie d'une auteure capable de jouer son rôle de passeuse, en nous faisant assumer nos rapports avec le passé et un présent toujours changeant, mais aussi avec l'Autre tout autant qu'avec nos semblables et nous-mêmes. 